NASATM - $7 q-208062$
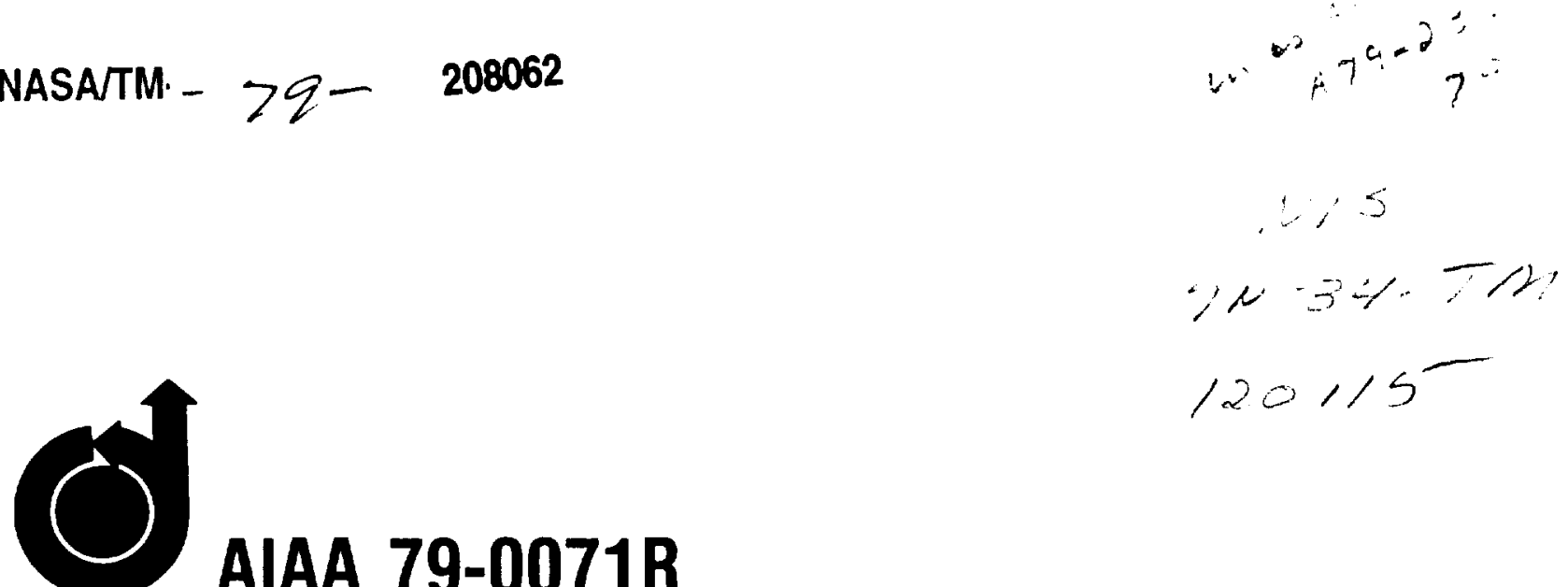

AIAA 79-0071R

Turbulence Modeling for Unsteady Transonic Flows

J. G. Marvin, L. L. Levy Jr., H. L. Seegmiller 


\title{
Turbulence Modeling for Unsteady Transonic Flows
}

\author{
J. G. Marvin, ${ }^{*}$ L. L. Levy Jr., † and H. L. Seegmiller $\ddagger$ \\ NASA Ames Research Center, Moffett Field, Calif.
}

\begin{abstract}
Conditionally sampled, ensemble-averaged velocity measurements, made with a laser velocimeter, were taken in the flowfield over the rear half of an $18 \%$ thick circular arc airfoil at zero incidence tested at $M=0.76$ and at a Reynolds number based on chord of $11 \times 10^{6}$. Data for one cycle of periodic unsteady flow having a reduced frequency $f$ of 0.49 are analyzed. A series of compression waves, which develop in the early slages of the cycle, strengthen and coalesce into a strong shock wave that moves toward the airfoil leading edge. A thick shear layer forms downstream of the shock wave. The kinetic energy and shear stresses increase dramalically, reach a maximum when dissipation and diffusion of the turbulence exceed production, and then decrease substantially. The response time of the turbulence to the changes brought about by the shock-wave passage upstream depends on the shock-wave strength and position in the boundary layer. The cycle completes itself when the shock wave passes the midchord, weakens, and the shear layer collapses. Remarkably good comparisons are found with computations that employ the time-dependent Reynolds averaged form of the Navier-Stokes equations using an algebraic eddy viscosity model, developed for steady flows.
\end{abstract}

\section{Nomenclature}

$a_{\infty}$

$c$

$C_{p}$

$f$

$\ell$

$M_{s}$

$M_{\infty}$

$n$

$P_{t}$

$\Delta p$

$R$

$R e_{c}$

$S$

$S C$

$S F$

$S P$

SHEAR

$t$

$t^{*}$

$T$ $=$ airfoil chord

$=$ pressure coefficient oscillation, $\pi f c / u_{\infty}$

$=$ reattachment

= separation

$=$ shock-wave passage $\Delta T$
= speed of sound in the freestream, $305 \mathrm{~m} / \mathrm{s}$

$=$ frequency of unsteady flow oscillation

$=$ reduced frequency of unsteady flow

$=$ Prandtl mixing length

$=$ Mach number ahead of the shock wave in a shock-fixed frame of reference

$=$ freestream Mach number

= number of instantaneous velocity samples used to form ensemble-averages

$=$ freestream total pressure

$=$ incremental pressure from the mean surface pressure

$=$ Reynolds number based on freestream conditions and airfoil chord

= shock-wave weakening and collapse

$=$ shock-wave formation

$=$ shear function, $-\left\langle u^{\prime} v^{\prime}\right\rangle / a^{2}$

$=$ time measured from the beginning of the oscillating pressure pulse

$=$ time measured from the beginning of the oscillating pressure pulse to the mean point of the ensemble-averaging interval

$=$ fractional time of one cycle of flow oscillation

Presented as Paper 79-0071 at the AIAA 17th Aerospace Sciences Meeting, New Orleans, La., Jan 15-17, 1979; submitted Feb. 13, 1979; revision received Sept. 28, 1979. This paper is declared a work of the U.S. Government and therefore is in the public domain. Reprints of this article may be ordered from AlAA Special Publications, 1290 Avenue of the Americas, New York, N.Y. 10019. Order by Article No. at top of page. Member price $\$ 2.00$ each, nonmember, $\$ 3.00$ each. Remittance must accompany order.

Index categories: Nonsteady Aerodynamics; Boundary Layers and Convective Heat Transfer-Turbulent; Transonic Flow.

*Branch Chief, Experimental Fluid Dynamics Branch. Associate Fellow AIAA.

†Research Scientist. Member AIAA.

$\ddagger$ Research Scientist.
$T_{r} \quad=$ time for ensemble-averaged velocity correlations to achieve their maximum value after shock passage

$\Delta T \quad=$ time interval used for ensemble averaging

TURBKE = turbulent kinetic energy function, $\left[\left\langle u^{\prime 2}\right\rangle+\left\langle v^{\prime 2}\right\rangle\right] / a_{\infty}^{2}$

$u, v \quad=$ velocity in the $x$ and $y$ directions, respectively

$u^{*}, v^{*} \quad=$ mean velocity in $x$ and $y$ directions, respectively during ensemble-averaging interval $\Delta T$

$u^{\prime}, v^{\prime} \quad=$ fluctuating velocity in $x$ and $y$ directions, respectively

$\langle u\rangle,\langle v\rangle \quad=$ ensemble-averaged velocity in $x$ and $y$ directions, respectively

$\left\langle u^{\prime 2}\right\rangle,\left\langle v^{\prime 2}\right\rangle=$ ensemble-averaged square of the velocity fluctuation in the $x$ and $y$ directions, respectively

$\left\langle u^{\prime} v^{\prime}\right\rangle \quad=$ ensemble-averaged fluctuation velocity correlation

$U \quad=$ normalized velocity, $\langle u\rangle / a_{\infty}$

$V \quad=$ total velocity, $\left(u^{2}+v^{2}\right)^{1 / 2}$

$\boldsymbol{x} \quad=$ distance from the airfoil leading edge in a direction along airfoil centerline

$y \quad=$ distance from the airfoil centerline in a direction normal to the centerline

$\bar{y} \quad=$ distance from the airfoil surface in a

$=$ boundary-layer thickness

Subscripts

$e \quad=$ edge of boundary layer

$m \quad=$ mean value

0 = upstream, ahead of shock wave

\section{Introduction}

TURBULENCE modeling is considered an important pacing item leading to successful development of computational fluid dynamics. ${ }^{1,2}$ Building-block experiments to guide the development and test the accuracy of modeling concepts have been initiated and are helping in the important area of turbulence model development. ${ }^{3,4}$ Although the modeling problem is by no means solved, progress has been demonstrated for several important classes of problems. For example, recent studies have shown that boundary-layer flows 
in the presence of moderate to large pressure gradients can be predicted adequately with scalar eddy viscosity models. ${ }^{5}$ Even flows with separation are predicted qualitatively and, in some instances, when separated zones are small, quantitatively 6,7 with eddy viscosity models; however, the details within the separated regions are not always correct.

One building-block experiment is the transonic flow over a circular arc airfoil. Depending on the freestream Mach number, the flow developed over the airfoil can be either steady with shock-induced separation extending beyond the trailing edge or unsteady with shock-induced separation. Several related experimental studies of this flow have been reported. ${ }^{8-10}$ Concurrent with these experimental studies, numerical simulations of the flowfield using the Reynolds averaged form of the Navier-Stokes equations have been undertaken. ${ }^{11-13}$ Although the details of the flows in the separated regions have not been predicted satisfactorily, all of the qualitative features have been.

With regard to the unsteady flow condition, Levy ${ }^{12}$ has shown that it is possible numerically to simulate most of the features in a qualitative sense. However, the predicted frequency of oscillation and the predicted magnitudes of the fluctuating surface pressures are $20 \%$ low and as much as $50 \%$ high, respectively. The question naturally arises as to whether these differences are attributable to the turbulence model employed and what improvements might be suggested after an experimental determination of the turbulence field. Little is known regarding turbulence in unsteady transonic flows. ${ }^{14}$ At lower speeds, where compressibility and pressure gradient effects are less severe, steady-flow turbulence models were employed in a quasisteady manner with remarkably successful results. ${ }^{\text {is }}$

In this paper, a detailed examination of the turbulent field in an usteady transonic flow undergoing shock-induced separation is presented. Ensemble-averaged mean and fluctuating velocities, obtained from conditionally sampled laser velocimeter data, are described and analyzed to assess the applicability of modeling concepts usually employed in steady-flow problems. Some comparisons with computations employing the Reynolds averaged Navier-Stokes equations with a mixing length turbulence model are then presented to illustrate the status of current predictive capabilities.

\section{Experimental Arrangement}

The test model was a circular arc airfoil with a chord of $20.34 \mathrm{~cm}$ and a ratio of thickness to chord of 0.18 . It was mounted in a rectangular test section with dimensions of $38.1 \times 25.4 \mathrm{~cm}$ and it spanned the shorter dimension. The upper and lower walls were contoured so that the supersonic region of the flow developed over the airfoil would not extend to the walls. Optical access for high-speed shadowgraph movies and the laser velocimeter tests was provided by glass windows, $14 \times 14 \mathrm{~cm}$, installed in the sidewalls. A combination of choking inserts and a translating wedge was used to establish the test Mach number. All test data were taken at $M_{\infty}=0.76$ and at a chord-based Reynolds number of $11 \times 10^{6}$ in a high Reynolds number blowdown facility.

Two beams from a 4-W argon laser were split, rotated \pm 45 deg, and intersected in the flowfield around the model near midspan. The resulting probe dimensions were approximately $0.3 \mathrm{~mm}$ in diameter by $3 \mathrm{~mm}$ in length in the spanwise direction. A two-channel synchronized counter system $^{10}$ was used to measure the velocities of $0.4-\mu \mathrm{m}$-diam polystyrene particles introduced into the flow in the settling chamber. These particles were assumed to follow the flow without significant lag. Corroborating evidence and computations supporting this assumption are noted in Ref. 10. The entire optical system was mounted on an optical bench and remote-controlled positioning was employed during test runs. About 2000 or more data samples were recorded at each spatial station. Instantaneous velocity components and surface pressure at midchord were recorded simultaneously on analog tape, digitized, and analyzed subsequently on a computer.

\section{Flow Domain Investigated}

A previous study established the Mach-Reynolds number domain of the flow characteristics around the airfoil in the present test section configuration. ${ }^{8}$ Depending on the MachReynolds number combination, the flow was either steady or unsteady. The case of interest for the present study is the unsteady periodic flow established at a Mach number of 0.76 and a Reynolds number of $11 \times 10^{6}$ where shock-induced separation occurred alternately $180 \mathrm{deg}$ out of phase on the upper and lower surface of the airfoil.

A sequence of shadowgraphs taken from a high-speed movie that depicts these flow characteristics for various fractions of the cycle is shown in Fig. 1. The movies were taken through glass windows that allowed viewing of the upper portions of the flow only. These reproductions of the shadowgraphs showing both sides are composites of frames from the same film, but with one image inverted and reversed; the phase difference in the flow, determined from a film timing-marker triggered by the same signal used to conditionally sample the velocity measurements, is taken into account. On the upper surface, a series of weak shock waves form near the trailing edge at early times, increase in strength, and coalesce into a single wave that moves toward the midchord. As the shock approaches midchord, it weakens appreciably, and the cycle repeats itself periodically about 185 times a second. A similar situation occurs on the lower surface 180 deg out of phase.

\section{Data Acquisition and Test Technique}

The periodic nature of the flow and phase relationship between the flow over the upper and lower surfaces of the airfoil were established previously. ${ }^{B}$ For the present in-
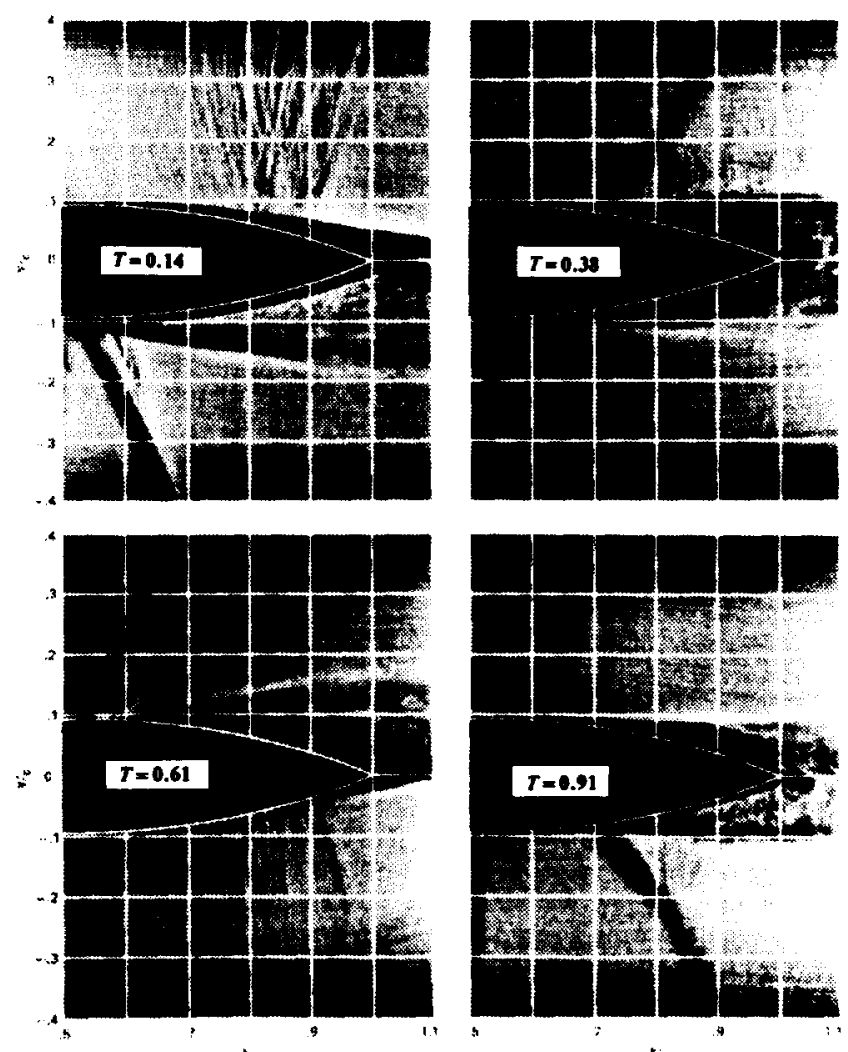

Fig. 1 Composite shadowgraphs showing the unsleady flowfield over the airfoil. $M_{\infty}=0.76, R_{c}=11 \times 10^{6}$. 


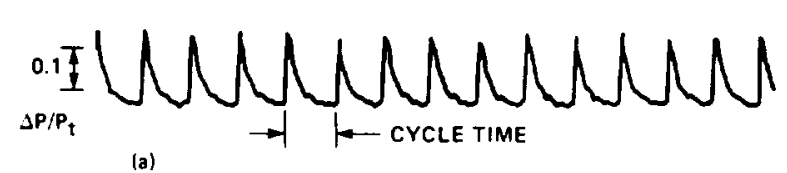

(a)

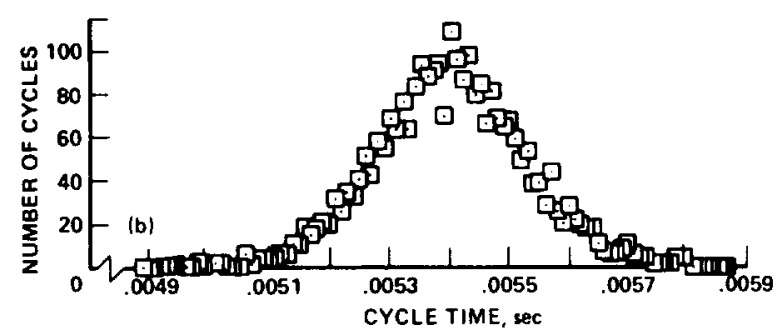

Fig. 2 Determination of periodicity of unsteady now. $M_{\infty}=0.76$, $R e_{c}=11 \times 10^{6}$. a) Pressure signal used to conditionally sample data. b) Distribution of flow oscillation cycle-times.

vestigation, the pressure time history of a fast-response transducer located at the midchord was used to define the period of the flow cycle as well as to provide the trigger for conditionally sampling of the velocity measurements.

A typical transducer signal output as a function of time is shown in the upper half of Fig. 2. The time response of the transducer reference volume, vented to the surface a small spanwise distance away, was large relative to that of the measurement volume, so the signal represents the change in pressure from the mean value established over many flow oscillations. For the purposes of this study the cycle time was chosen as the time between successive steep rises in the pressure signal, which corresponds with the passage of the shock wave upstream. An electronic circuit designed to trigger on the steep rise rate of the pressure was used to determine the time of each cycle and to provide a means for conditionally sampling the laser data.

A typical distribution of cycle times is shown in Fig. 2 to illustrate the periodicity of the flow. In the example, over 2000 flow oscillations occurred. The distribution is Gaussian and the mean cycle time was $5.40 \mathrm{~ms}$ with a standard deviation of $0.132 \mathrm{~ms}$ or $\pm 2.4 \%$. The randomness about the mean is attributable to both the uncertainty in trigger time and to the flow randomness, but the randomness associated with the trigger time is rather small compared to the standard deviation, about $\pm 0.2 \%$.

The dual counter data rate was of the order of a few thousand per second or approximately 5 per flow cycle and so the data from many cycles were combined to obtain the velocity for a single cycle by conditionally sampling the data on the basis of a time beginning with the onset of the transducer pressure rise. In order to account for the randomness of the cycle times, each velocity measurement time was normalized by the actual cycle time during which the velocity data were acquired. An example of the conditionally sampled data for a single point in the flowfield is presented in Fig. 3. (Note that the velocity scale is broken to provide an expanded scale.) The two synchronous velocity components have been combined to give the total velocity which is shown as a function of the dimensionless fraction of time $T$ for a single cycle of oscillation. Over 2000 data samples were used to construct this plot. The velocity increases with time and then shows a marked decrease as the shock wave passes upstream.

An ensemble-average of the conditionally sampled data was then performed to obtain mean velocity, turbulent kinetic energy, and turbulent shear stress for various times within the cycle. Consider the velocity data in Fig. 3 to be typical of the individual components $u$ and $v$. The ensemble-averaging time $\Delta T$ must be long relative to the characteristic time of the turbulence and short relative to the characteristic cycle time. For $\Delta T<1$ and considering the general case where $\langle u\rangle$ can change with time over the averaging interval, the velocity to first order can be expressed as

$$
u(t)=\left\langle u^{*}\right\rangle+\frac{\left.\mathrm{d}<u^{*}\right\rangle}{\mathrm{d} t^{*}}\left(t-t^{*}\right)+u^{\prime}(t)
$$

where the first two terms represent the ensemble-averaged velocity at any time $t$ within the interval $\Delta T$, and $t^{*}$ represents the ensemble-averaged time for the interval $\Delta T$. Expressions for the conditionally sampled, ensemble-averaged velocities and their correlations for the interval $\Delta T$ were determined from $\mathrm{Eq}$. (1). They become

$$
\begin{aligned}
& <u(t)>=<u^{*}>=\frac{I}{n} \sum_{i=1}^{n} u_{i} \\
& \langle v(t)\rangle=\left\langle v^{*}\right\rangle=\frac{1}{n} \sum_{i=1}^{n} v_{i} \\
& \left\langle u^{\prime}(t) v^{\prime}(t)\right\rangle=\left\langle u^{\prime} v^{\prime}\right\rangle \\
& =\frac{I}{n} \sum_{i=1}^{n}\left\{\left[u_{i}-\left(<u^{*}\right\rangle+\frac{\left.d<u^{*}\right\rangle}{\mathrm{d} t^{*}}\left(t_{i}-t^{*}\right)\right]\right. \\
& \times\left[v_{i}-\left(\left\langle v^{*}\right\rangle+\frac{d\left\langle v^{*}\right\rangle}{\mathrm{d} t^{*}}\left(t_{i}-t^{*}\right)\right]\right\} \\
& \left\langle u^{\prime}(t) u^{\prime}(t)+v^{\prime}(t) v^{\prime}(t)\right\rangle=\left\langle u^{\prime 2}\right\rangle+\left\langle v^{\prime 2}\right\rangle \\
& =\frac{I}{n} \sum_{i=1}^{n}\left\{\left[u_{i}-\left(<u^{*}\right\rangle+\frac{\mathrm{d}\left\langle u^{*}\right\rangle}{\mathrm{d} t^{*}}\left(t_{i}-t^{*}\right)\right)\right]^{2} \\
& \left.\left.+\left[v_{i}-\left(<v_{i}^{*}\right\rangle+\frac{d<v^{*}>}{\mathrm{d} t^{*}}\left(t_{i}-t^{*}\right)\right)\right]^{2}\right\}
\end{aligned}
$$

where $n$ is the total number of velocity samples in the interval $\Delta T$.

The averaging interval $\Delta T$ was chosen as 0.05 . This interval corresponds to a real time that is longer than the characteristic time scale of the turbulence, $\delta / u_{e}$, by factors of 3 and 7 , respectively, depending on the maximum and minimum overall mean velocity and length scale variations throughout the cycle. Further, the interval is sufficiently small to insure that the variation of mean velocity over the interval can be given with good confidence by the first-order correction in Eq. (1). It is important to note that the present technique employing conditional sampling does not require corrections to the equations for ensemble averages to account for particle sampling bias of the type discussed in Ref. 16. Also, the present technique is not restricted to the choice of the averaging interval in the same way as a hot wire. In the hot wire case, the maximum interval determines the largest scale of the turbulence that can be observed; in the present case, scales for all frequencies can be obtained provided that 1) the sampling takes place over enough cycles to capture them, and 2) the probabilities of particles being present in large- and small-scale turbulence are equal.

Conditional sampling as applied in the present investigation may introduce uncertainties in the ensemble averages due to the randomness in the cycle times discussed previously. Most of this uncertainty was accounted for by normalizing the sampling times by the actual cycle time during which the data were acquired. The validity of this procedure is substantiated by the fact that outside the viscous regions, except in some special instances discussed later and attributed to flow 
physics, the ensemble-averaged shear stresses approach zero as expected, and the turbulent intensities tend toward their expected freestream levels.

The number of data samples used to compile the ensemble averages varied as a result of test technique. Usually the number of samples $n$ was more than 100, although for some locations $n$ was less. The effects of the sample number on measurement error can be estimated as indicated in Ref. 17. For an assumed confidence limit, the uncertainty in velocity depends on the sample number and the turbulent intensity whereas the error in turbulent intensity depends only on the sample number. Using the analysis of Ref. 17, the range of estimated errors in velocity and turbulent intensity for the present data, based on a $95 \%$ confidence limit, lies between a few percent and $20 \%$, with the larger error occurring where the velocity was smallest and where the turbulent intensity relative to that velocity was largest. No estimate was made of the errors in shear stresses, but they probably are in the same range.

\section{Results and Discussion}

\section{Experimental Observations}

Profiles of conditionally sampled, ensemble-averaged axial velocity, turbulent shear stress, and turbulent kinetic energy were obtained at eight axial stations between $x / c=0.6$ and 1.05. A movie depicting the changes in these profiles with time was made and used with the shadowgraph movie to interpret the results as follows.

At early times within the cycle, the boundary layer is thin and the flow is attached over the upper airfoil surface. As the shock strengthens and moves forward the boundary layer thickens and reversed flow occurs in the vicinity of $x / c=0.8$ and at the trailing edge. As time increases further and the shock continues to move upstream, the reversed flow zone increases in size. At the time the weakened shock moves near $x / c=0.5$, the separation collapses, and the shear layer thins. Other noteworthy observations are a significant increase in the turbulent kinetic energy in the shear layer when the shock wave passes by the measuring location and the presence of "negative" shear subsequent to the weakening and collapse of the shock wave and during the formation of the series of compression waves. Apparently, the compression waves have a bearing on this observation as the "negative" shear stresses occur near the edge or outside of the boundary layer when these waves are observed.

Time histories of the ensemble-averaged data for three chord stations and various locations above the airfoil surface are shown in Fig. 4. In the upper portions of the figure, the

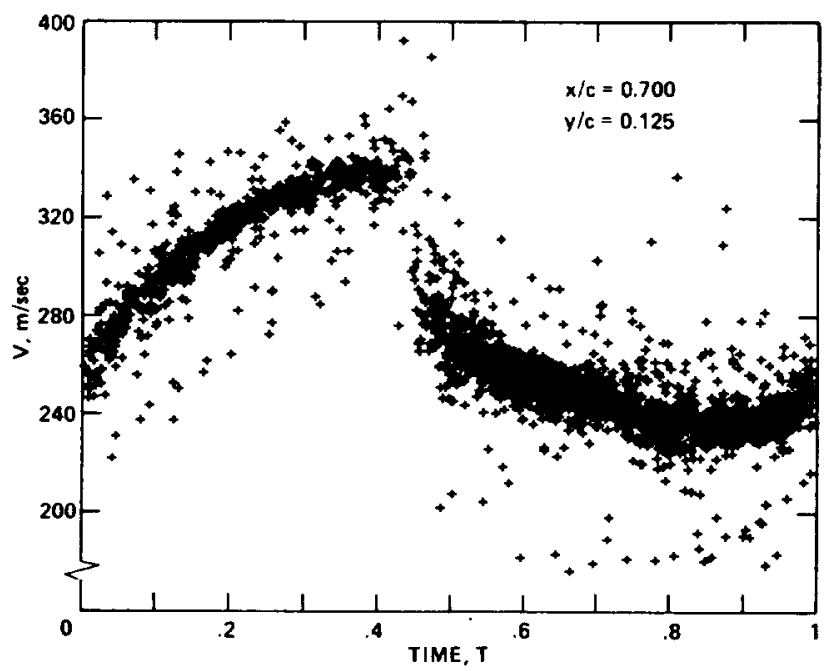

Fig. 3 Time history of velocity from conditionally sampled dala for one cycle of now oscillation. $M_{\infty}=0.76, R e_{c}=11 \times 10^{6}$. axial velocity is shown for all measuring stations up to $y / c=0.15$. In the remaining portions of the figure, only the data below the boundary-layer edge are displayed. This was done for clarity because the boundary-layer thickness varies considerably with time during the flow oscillation and many of the measuring points are outside the boundary layer at early time and not essential to the discussion on modeling that follows. The notations along the time axis refer to shock formation $(S F)$, shock passage $(S P)$, the beginning and end of significant separation ( $S$ and $R$ ), and shock collapse $(S C)$. The shock formation time was arbitrarily taken as the time when the series of compression waves present at early times coalesced into a single strong wave; the shock passage coincided with the passage of the leading edge of the shock foot; and the zone of separation was inferred from the velocity histories.

The time histories show that, after the strong shock formation and shock passage upstream, the velocities in the inner portions of the boundary layer are retarded more than in the outer portions; the corresponding turbulent kinetic energy and shear increase, maximize, and diminish. At two locations, $x / c=0.85$ and 0.95 , the shear during the initial portion of the cycle is negative; this observation will be discussed subsequently. As the shock wave moved past a measuring station, the velocities in the outer, inviscid regions of the flow adjusted to values consistent with the passage of an oblique wave. An example of this adjustment is given for $x / c=0.7$ and $y / c=0.125$. This estimate, taken from Ref. 10, was made using the velocity data upstream of the shock and the shock angle measured from shadowgraphs.

The shadowgraphs and velocity time histories were used to estimate shockwave position and zones of significant separation. The results are shown in Fig. Sa. The magnitude of the Mach number ahead of the shock wave, in a shockfixed frame of reference, is shown in Fig. 5b. The zone of significant separation was defined where at each chord station the first measuring point above the surface indicated zero velocity. This height ranged from between 10 and $20 \%$ of the boundary-layer thickness. Actual separation and reattachment would occur beyond the extent of this zone. In the early portions of the cycle, the shock wave moves upstream more rapidly than during the later portions. Significant shock-induced separation occurs subsequent to shock passage.

The foregoing data provide means for evaluating computations of unsteady transonic flows with shock-induced separation and details of the turbulent flow that should help in the development of adequate turbulence models. In regard to the latter, it is convenient to divide the discussion in two parts, the first dealing with the portion of the cycle between the time the shock weakens and the shear layer collapses until the shock forms subsequently $(S C<T<S F$ ), and the second dealing with the remaining portion of the cycle $(S F<T<S C)$.

As the shock wave weakens and approaches midchord, the shear layer collapses and it takes about $15 \%$ of the cycle time for large-scale turbulence to pass downstream over the airfoil. The turbulent kinetic energy decreases, but at some of the axial locations the shear inside the outer two-thirds of the boundary layer reverses sign and becomes negative as shown in Fig. 4. At these axial locations there were usually other locations outside the boundary layer that also had negative shear stresses. The presence of negative turbulent shear stresses in the outer regions of the boundary layer and above has been observed in other experiments ${ }^{18,19}$ for steady flows in the presence of severe adverse pressure gradients. At first, it was believed that in the present study these negative shears were caused by the downward motion of the flow after the shear layer collapsed. To verify this conjecture, the strain rates $(\partial u / \partial y+\partial v / \partial x)$, at the measurement points in the flowfield were evaluated. The strain rate was positive for all $y / c$ locations during the period, $S C<T<S F$. Thus, the 

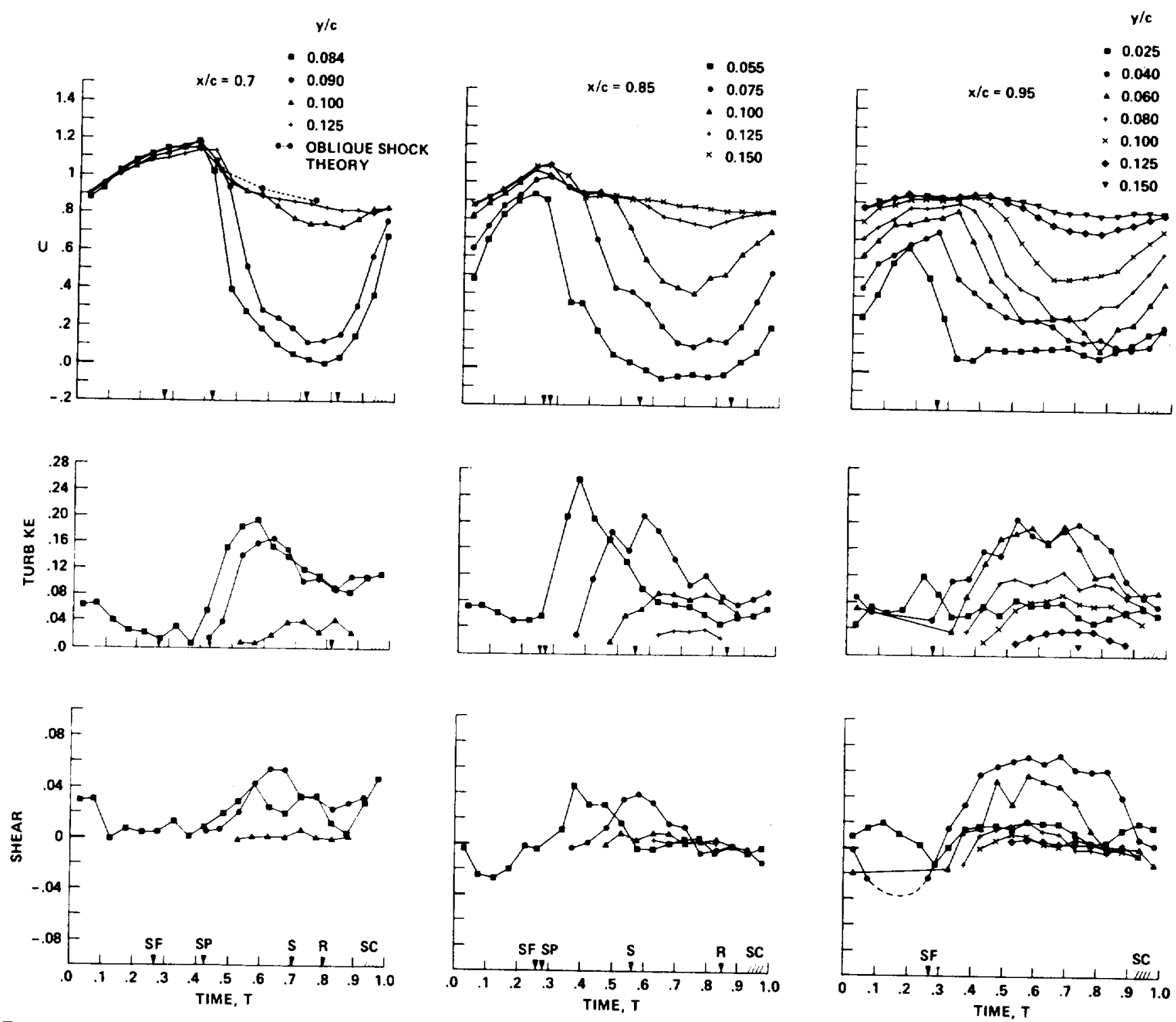

Fig. 4 Time histories of ensemble-averaged velocity, turbulent kinetic energy, and turbulent shear stresses for a cycle of oscillation. $M_{\infty}=0.76$,
$R e_{c}=11 \times 10^{6}$.

turbulent shear was not always directionally in phase with the mean flow strain rate. It is believed that the negative shear stresses occur as a result of the interaction of the boundary layer with a series of unsteady compression waves that form after the shear layer collapses and passes downstream. The presence of these waves may also explain why measureable shear stresses were present outside the apparent edge of the boundary layer. In the strict sense, scalar eddy viscosity formulations would not appropriately model this observation since the shear would not be proportional to the strain rate. However, it is felt that, since this observation is restricted to the outer portions of the boundary layer and beyond, it would have little influence on predictions of boundary-layer development during this portion of the cycle.

Attention is now focused on the time interval between strong shock-wave formation and collapse, $S F<T<S C$. The velocities shown in Fig. 4 decrease during the formation of the shear layer aft of the shock wave and rise toward the end of the time interval when the the layer begins to thin. The inner portions of the layer have lower momentum and respond more to the change in flow conditions brought about by the presence of the shock wave. The turbulent kinetic energy and shear stress increase to a maximum, at which time dissipation and diffusion of the turbulence overcome production. Thereafter they decrease and the shear becomes small and goes negative in the separated regions of the flow where the velocities and corresponding velocity gradients become negative. In general, the shear appears to be in phase with the kinetic energy, each reaching its maxima and minima at approximately the same cycle time.

Although there is a deceleration of the flow after shock passage, it is informative to view the rise time for reaching maximum turbulent kinetic energy (and/or shear stress) for $x / c \leq 0.85$ as an equilibration time for turbulence production in response to the changing conditions imposed by the shock wave. (It should be noted that the actual time for the shock wave to pass the measuring location and the time for seed particles to adjust to within $99 \%$ of the velocity jump across the wave are both an order of magnitude smaller than the time interval between data points in the time histories.) The time to reach maximum turbulent kinetic energy and shear depends on the shock-wave strength. For example, at $x / c=0.7$, where the Mach number ahead of the shock wave shown in Fig. $5 \mathrm{~b}$ is maxiumum, the time for the turbulent kinetic energy to reach a maximum is about $t$ wice as long as at $x / c=0.85$. Viewed in a shock-fixed frame of reference, the number of upstream boundary-layer thicknesses for adjustment, $u_{m} T_{r} / \delta_{0}$, is between 20 and 60 for $x / c=0.85$ and 0.7 , respectively, where $u_{m}$, an average speed over the rise time $T_{r}$, was chosen as 0.6 $u_{e}$. 

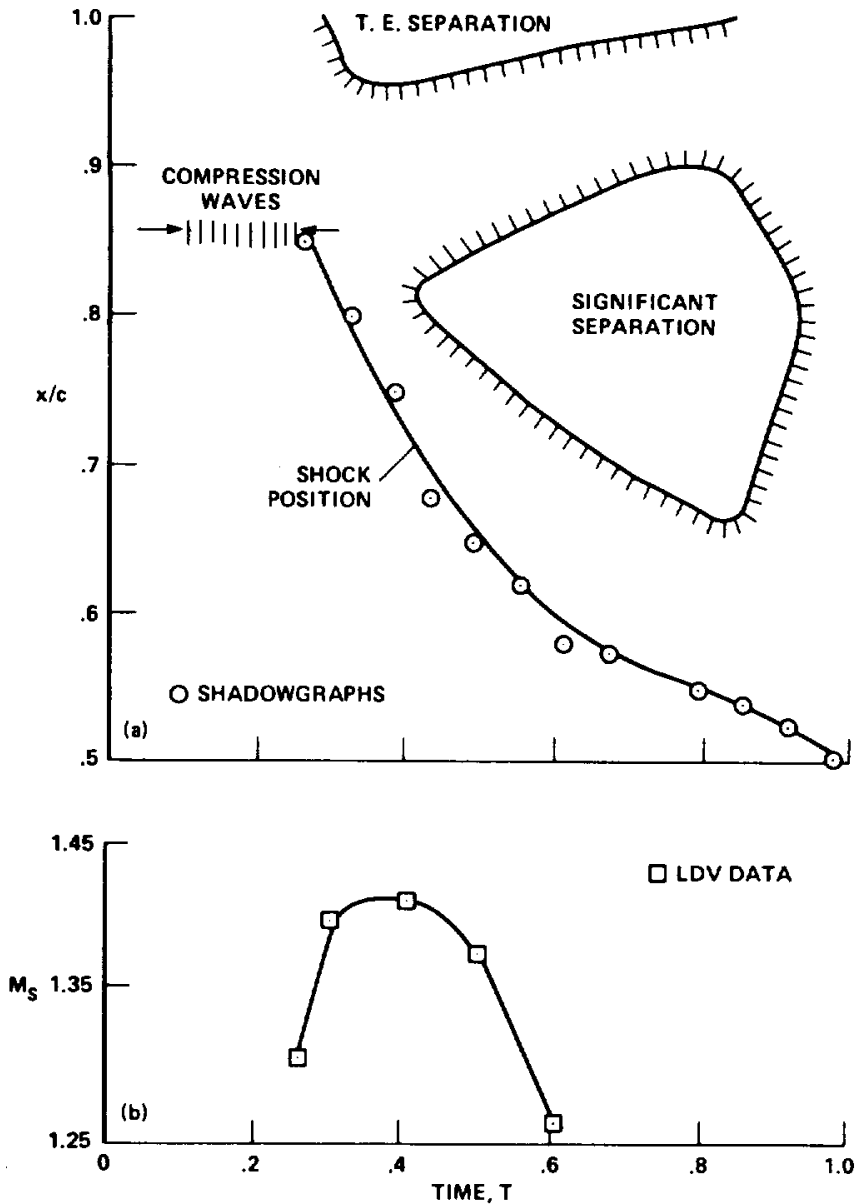

Fig. 5 Flowfield characteristics for a cycle of now oscillation determined from shadowgraphs and ensemble-averaged velocity data. $M_{\infty}=0.76, R e_{c}=11 \times 10^{6}$. a) Shock-wave position and zones of significant separation, b) Mach number ahead of shock wave.
EXPERIMENT (SHADOWGRAPHS)
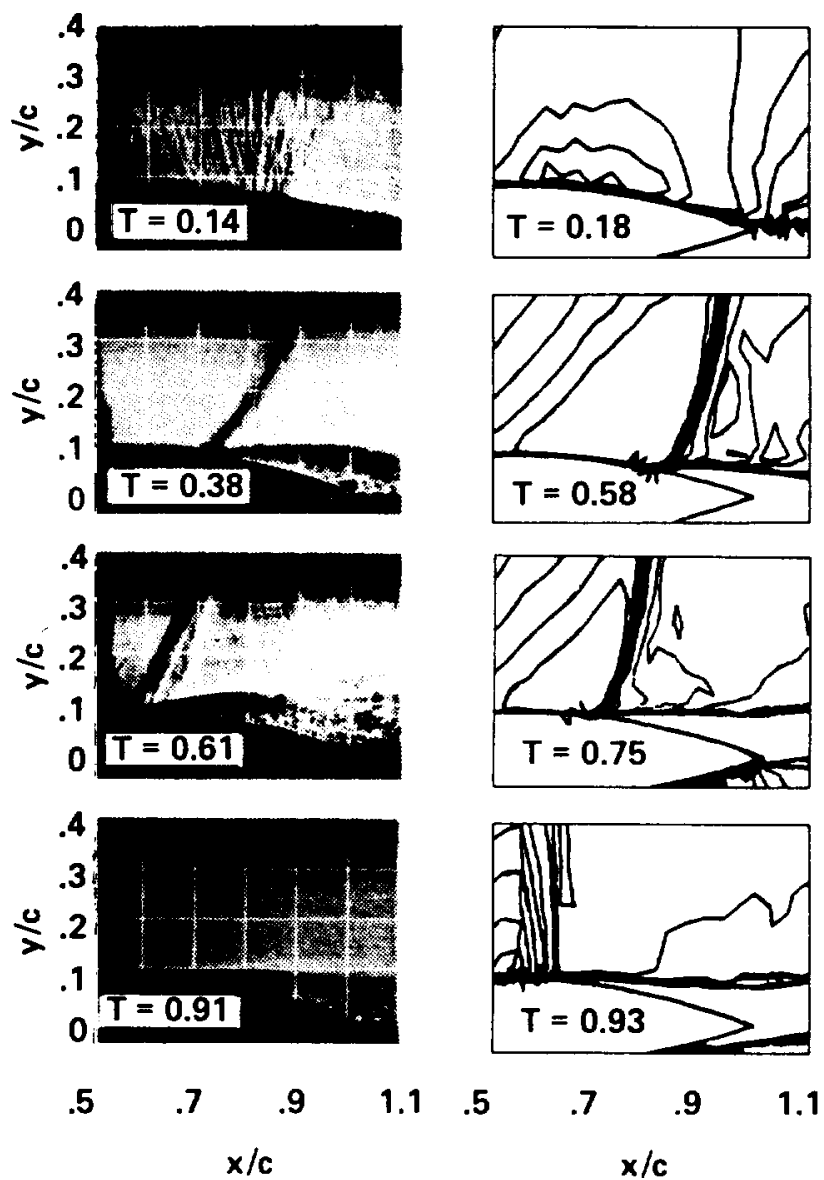

Fig. 6 Comparison of shadowgraphs with Mach contours from the computations. $M_{\infty}=0.76, R e_{c}=11 \times 10^{6}$.
COMPUTATION (MACH CONTOURS)
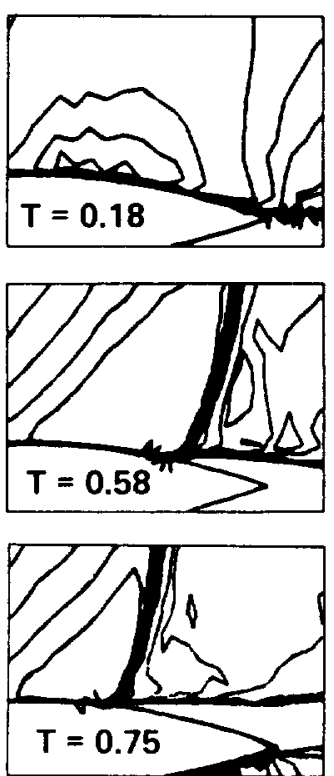

1

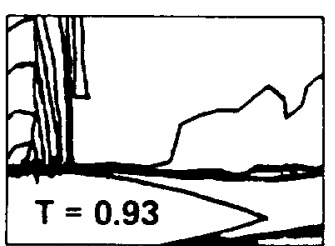

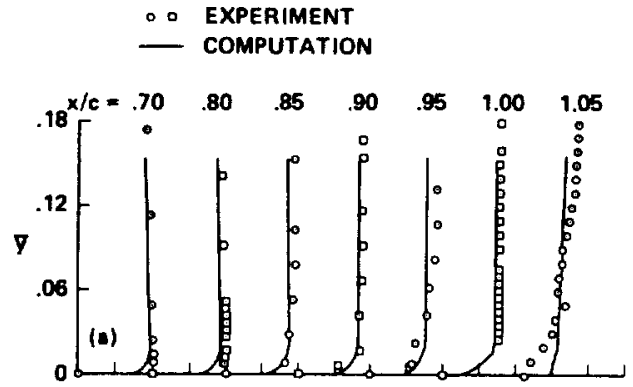
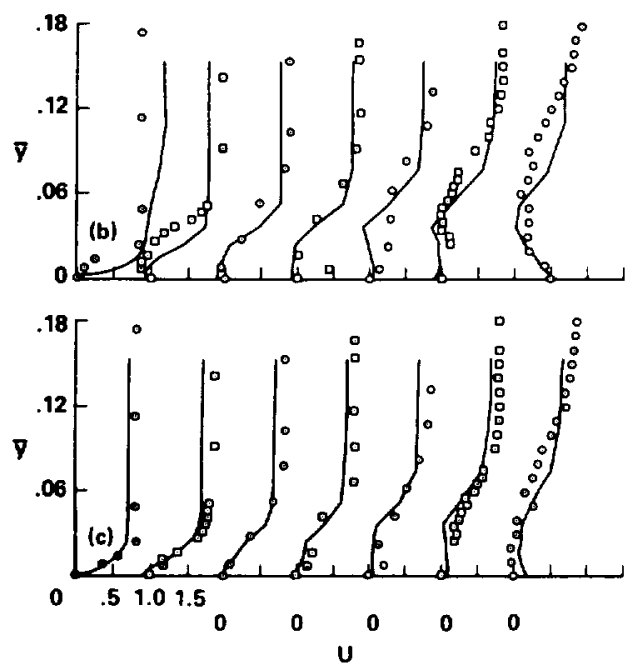
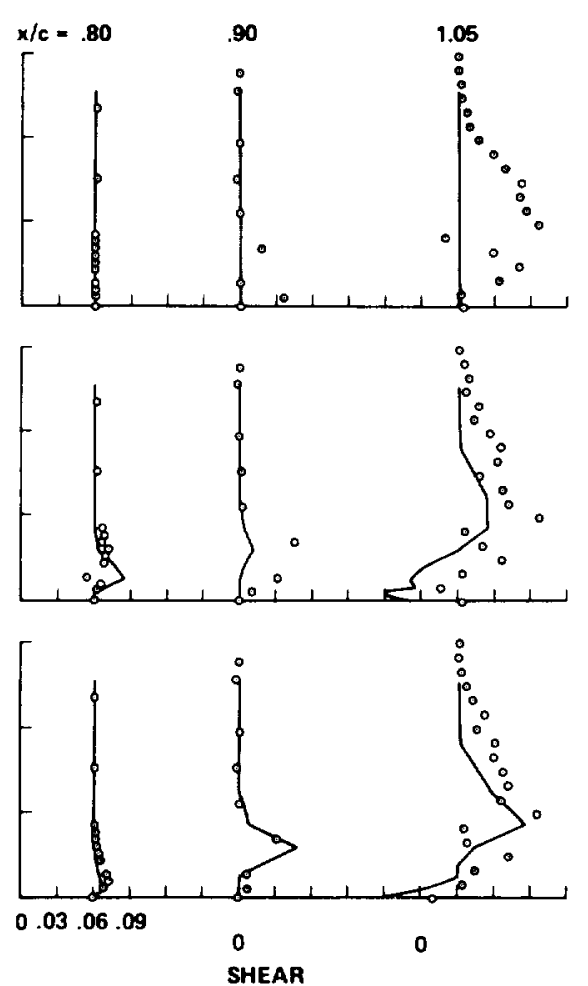
Fig. 8 Comparison of velocity and shear stress time histories from the experiment and computations. $M_{\infty}=0.76, R e_{c}=11 \times 10^{6}$.
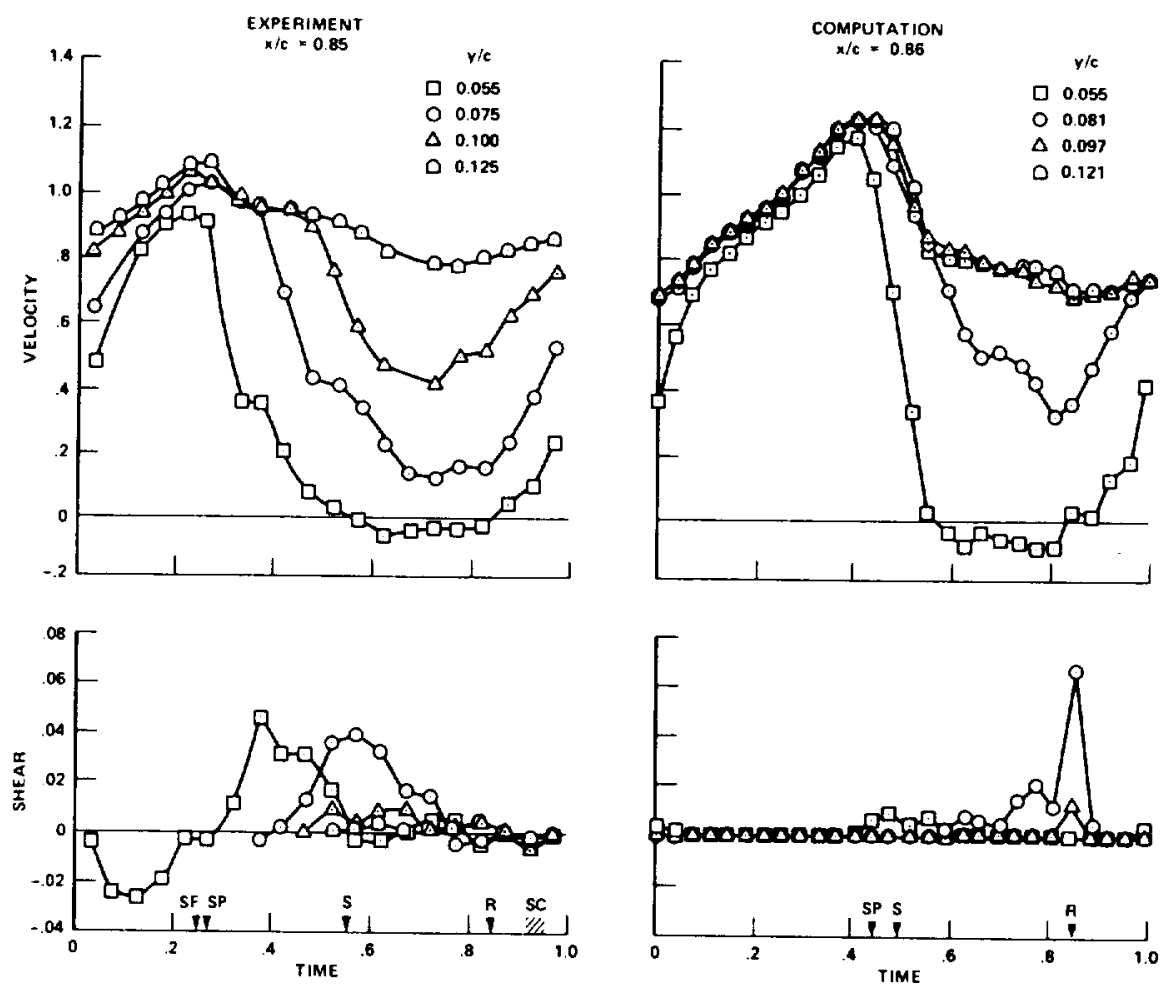

\section{Comparisons with Computations}

Computations of this unsteady flow using the time dependent Reynolds averaged form of the Navier-Stokes equations were first reported in Ref. 12. A primitive turbulence model developed for steady flows was employed. An algebraic mixing length model was applied outside the region bounded by the dividing streamline that separated the main and recirculating regions of the flow. Inside the recirculating region, the eddy viscosity model was varied linearly between the value at the dividing streamline and zero at the airfoil surface. (See Refs, 10 and 12 for details of the turbulence model.) Over the separated region, the mixing length was frozen at the value achieved prior to separation in an attempt to account for the lag in adjustment of the turbulence to the changing conditions imposed by the shock wave. It was previously shown ${ }^{10,12}$ that the computations reproduce the unsteady features of the flow, but that the reduced frequency of oscillation was low by about $20 \%$ and that the pressures downstream of the shock wave were too high, in some cases double the values of the experiment. Additional comparisons with the experiment are made next.

The shock-wave shapes from the shadowgraphs are compared with computed Mach number contours in Fig. 6. Although all the flow features were reproduced by the computation, they occurred later in the cycle so the comparisons are made at different $T^{\prime} s$. The computed shock wave strengthens, moves forward, and weakens substantially, in agreement with experimental observations. However, the predicted shock wave is more normal and the pressures downstream of the shock wave are greater. Similar features, $180 \mathrm{deg}$ out of phase, occur on the lower half of the airfoil just as in the experiment.

Computed velocity and shear stress profiles are compared with some of the experimental profiles in Fig. 7. (The times for the computations are shifted for the reason mentioned previously.) At the earliest time the velocity profiles agree reasonably well but the experimental shear profiles show a residual of the previous cycles' thick layer near and aft of the trailing edge that is not apparent in the computations. At the intermediate time, when the shock wave has passed to a position upstream, both the experiment and computation show regions of reversed flow, reattachment, and a sub- stantial thickening of the shear layer. However, the location and extent of the separated region differ somewhat from the experiment, as does the maximum shear stress achieved in the shear layer. At the latest time the velocity and shear profiles are in good agreement.

The shadowgraph and profile comparisons were made at different times to illustrate that the computations reproduce most of the measured features of the flow. Comparisons of the time histories were also made for each experimental axial location, and an example is given in Fig. 8 for $x / c=0.85$. Although over 20 values of $y / c$ are used in the computation across the shear layer, only those near the experimental locations are plotted to facilitate comparison. Locations of shock passage, and the separation zone at the surface for the computation are shown on the abscissa. The computations have all the same features of the experiment, but the velocity before shock passage is somewhat higher and the change in velocity in the outer portion of the flow is greater due to the presence of a more normal shock wave, as mentioned before. The adjustment of the velocities to the change in conditions imposed by the shock wave at different positions in the layer is very much the same as in the experiment. The response of the shear stress to shock passage also shows the same behavior exhibited by the experiment, but the magnitude is generally smaller, except at $T=0.85$ where there is a sudden increase where reattachment occurred and where the turbulence model formulation changed.

The computations, using a rather simple turbulence model, developed for steady-flow applications reproduced all the features of this unsteady flow with shock-induced separation. The implication is that the model does not have to be a precise one that reproduces all the fine details of the turbulent structure, but rather one that can account adequately for turbulence production and destruction mechanisms. In both the steady and unsteady computations, a lag to turbulence adjustment was incorporated by freezing the mixing length at its value ahead of separation. It was found experimentally that this lag depended on both shock-wave strength and position in the boundary layer. Therefore, an improvement could be expected with higher-order models which automatically build in these lags through the use of additional differential equations for the turbulence dissipation. ${ }^{6}$ The 
experimental data on turbulent shear stress and turbulent kinetic energy presented herein may help in determining more appropriate modeling constants in the higher-order models for the steady flow case. For example, the data could be employed to help optimize solutions to the unsteady form of the modeling equations for turbulent kinetic energy and dissipation using the experimental velocity field and gradients as known input.

\section{Conclusions}

The unsteady flowfield around a circular arc airfoil undergoing shock-induced separation was studied. A two-color, dual-channel laser velocimeter was used to measure the instantaneous velocities for a region of the flow extending from midchord to a location beyond the trailing edge. Advantage was taken of the periodic nature of the unsteady flow in order to conditionally sample the data over many cycles of flow oscillation so that the velocity field over a single cycle could be reconstructed. Ensemble averages were then taken over a time interval that was short relative to the total cycle time in order to evaluate the mean velocity, turbulent kinetic energy, and shear stresses. During the initial portion of the cycle, the flow on the upper surface of the airfoil was attached and accelerating. As the speed increased, a series of compression waves strengthened and coalesced into a single shock wave that moved upstream. A thick shear layer developed downstream of the shock wave and flow separation was detected. As the shock wave approached midchord, it weakened and the shear layer collapsed and was convected downstream. On the other side of the airfoil, a similar sequence occurred $180 \mathrm{deg}$ out of phase.

Time histories of the data show that the turbulent kinetic energy and shear stress downstream of the shock wave increased dramatically. The time required for each to reach its maximum level depended on the shock-wave strength and the position in the boundary layer. Thereafter, dissipation and diffusion of turbulence exceeded production and the kinetic energy and shear stress decreased. It was not possible to find an algebraic length scale that correlated with shear-layer thickness, nor was the ratio of shear stress to turbulent kinetic energy constant; however, the shear appeared to be in phase with the kinetic energy because the times to achieve maxima and minima were about the same.

Comparisons of the measurements with computations employing the time dependent, Reynolds averaged form of the Navier-Stokes equations with a primitive eddy viscosity model developed for steady flows showed that all the features of the flow were predicted. The implication seems to be that turbulence models developed for steady flows apply and that the model need not reflect all the fine details of the turbulent structure but rather account in an approximate way for the production and destruction of the turbulence.

\section{References}

'Bradshaw, P., “Review-Complex Turbulent Flows," Transactions of the ASME Journal of Fluids Engineering, Vol. 97, June 1975, pp. 146-154.
${ }^{2}$ Chapman, D.R., Mark, H., and Pirtle, M.N., "Computers vs. Wind Tunnels for Aerodynamic Flow Simulation," Aeronautics and Astronautics, Vol. 13, April 1975, pp. 22-30.

${ }^{3}$ Marvin, Joseph G., "Experiments Planned Specifically for Developing Turbulence Models in Computations of Flow Fields Around Aerodynamic Shapes," AGARD Conference Proceedings No. 210 on Numerical Methods and Wind-Tunnel Testing, June 1976.

${ }^{4}$ Marvin, Joseph G., "Turbulence Modeling for Compressible Flows," NASA TM X-73, 188, Jan. 1977.

${ }^{5}$ Horstman, C.C., Kussoy, M.I., and Lanfranco, M.J., "An Evaluation of Several Compressible Turbulent Boundary-Layer Models: Effects of Pressure Gradient and Reynolds Number," AIAA Paper 78-1160, Seattle, Wash., July 1978.

${ }^{6}$ Viegas, John R. and Horstman, Clifford C., "Comparison of Multiequation Turbulence Models for Several Shock Separated Boundary-Layer Interaction Flows," AIAA Paper 78-1165, Seattle, Wash., July 1978.

${ }^{7}$ Coakley, T.J., Viegas, J.R., and Horstman, C.C., "Evaluation of Turbulence Models for Three Primary Types of Shock Separated Boundary Layers," AIAA Paper 77-692, Albuquerque, N. Mex., June 1977.

${ }^{8}$ McDevitt, J.B., Levy, L.L., Jr., and Deiwert, G.S., "Transonic Flow About a Thick Circular-Arc Airfoil," AIAA Journal, Vol. 14, May 1976, pp. 606-613.

${ }^{9}$ Rubesin, M.W., Okuno, A.F., Levy, L.L., Jr., McDevitt, J.B., and Seegmiller, H.L., "An Experimental and Computational Investigation of the Flow Field About a Transonic Airfoil in Supercritical Flow With Turbulent Boundary-Layer Separation," 10th Congress of the International Council of the Aeronautical Sciences, Ottawa, Canada, Oct. 1976; also NASA TM X-62, 465, July 1976.

${ }^{10}$ Seegmiller, H.L., Marvin, J.G., and Levy, L.L., Jr., "Steady and Unsteady Transonic Flows," AIA A Journal, Vol. 16, Dec. 1978, pp. $1262-1270$.

"Deiwert, G.S., "Computation of Separated Transonic Turbulent Flows," AIAA Journal, Vol. 4, June 1976, pp. 735-740.

${ }^{12}$ Levy, L.L., Jr., "Experimental and Computational Steady and Unsteady Transonic Flowfields about a Thick Airfoil," AIAA Journal, Vol. 16, June 1978, pp. 564-572.

${ }^{13}$ Coakley, T.J. and Bergman, M., "Effects of Turbulence Model Selection on the Prediction of Complex Aerodynamic Flows," AIAA Paper 79-0070, New Orleans, La., Jan. 1979.

${ }^{14}$ McCroskey, W.J., "Introduction on Unsteady Aspects of Separation in Subsonic and Transonic Flow," Paper 6, AGARD Lecture Series No. 94, Three Dimensional and Unsteady Separation at High Reynolds Numbers, Von Karman Institute, Rhode-SaintGenese, Belgium, Feb. 1978.

${ }^{15}$ Cousteix, J. and Houdeville, R., "Turbulent Boundary Layer Calculations in Unsteady Flow," Communication presentée a la Conference sur les Methodes Numeriques et Dynamique des Fluides Appliquee, Reading University, G.B., Jan. 1978.

${ }^{16}$ Dimotakis, P.E., "Single Scattering Particle Laser Doppler Measurements of Turbulence," Applications of Non-Intrusive Instrumentation in Fluid Flow Research, AGARD Conference Proceeding No. 193, Saint-Louis, France, May 3-5, 1976.

${ }^{17}$ Yanta, W.J., "Turbulence Measurements With a Laser Doppler Velocimeter," Ph.D. Thesis, The Catholic University of America, Washington, D.C., 1973.

${ }^{18}$ Kussoy, M.I., Horstman, C.C., and Acharya, M., "An Experimental Documentation of Pressure Gradient and Reynolds Number Effects on Compressible Turbulent Boundary Layers," NASA TM-78488, 1978

${ }^{19}$ Mateer, George G. and Brosh, Aviel, "A Normal Shock-Wave Turbulent Boundary-Layer Interaction at Transonic Speeds," AlAA Paper 76-161, Washington, D.C., Jan. 1976. 